\title{
VIBRATION ANALYSIS OF THE OSCILLATION SUPPORT OF COLUMN LOAD CELLS IN LOW SPEED AXLE-GROUP WEIGH-IN-MOTION SYSTEM
}

\author{
Lai Zhengchuang ${ }^{1,2}$, Yang Xiaoxiang ${ }^{1,3}$, Yao Jinhui ${ }^{1,2}$ \\ ${ }^{1}$ College of Mechanical Engineering and Automation, Fuzhou University, Fuzhou, China, lzcfjjl@126.com \\ ${ }^{2}$ Fujian Key Laboratory of Force Measurement (Fujian Institute of Metrology), Fuzhou, China, lzcfjjl@126.com \\ ${ }^{3}$ Quanzhou Normal University, Quanzhou, China, yangxx@fzu.edu.cn
}

\begin{abstract}
:
Under the condition of dynamic weighing, the support types of the column load cells can be divided into elastic support and oscillation support. The weighing system with oscillation support shows significant vibration characteristics, which affects the weighing accuracy and the fatigue life of the load sensor. In this paper, the dynamic characteristics of the oscillation supporting column load cell in low speed axle-group weigh-in-motion system are analysed. It is found that the restoring force of the oscillation support is approximately proportional to the oscillation angle and the applied vertical load. The dynamic equation of the oscillation support vibration of the column load cells established by means of d' Alembert principle. The numerical calculations of the dynamic response of the weighing system with oscillation support are carried out in the free state and dynamic weighing state respectively. The factors affecting the amplitude and recovery time of the support vibration are obtained. This study provides a reference for the analysis of the dynamic weighing accuracy.
\end{abstract}

Keywords: vibration and wave; oscillation support; vibration analysis; dynamic weighing

\section{INTRODUCTION}

Column load cell is an important component of the axle group dynamic truck scale. Under the condition of dynamic weighing, the support types of the column load sensors can be divided into elastic support and oscillation support. The weighing system with oscillation support shows significant vibration characteristics, which affects the weighing accuracy and the fatigue life of the load cells [1] [2].

In this paper, the force analysis is carried out for the weighing process of the oscillation support column load cell. In the dynamic weighing system, the low speed axle group weigh-in-motion (LSWIM) system is used as the research object to analyse the axle load conditions. The dynamic response of the weighing system is obtained through dynamic system model and simulation.

\section{OSCILLATED STABILITY ANALYSIS OF THE LOAD CELL}

Oscillated support column load cell consists of strained elastomer and an upper and lower indenter. The lower indenter is fixed on the foundation and is in point-surface contact with the strained elastomer. The upper and lower ends of the elastomer are spherical and can roll on the upper and lower indenters. The upper indenter is fixedly connected to the weighing platform, the bottom surface is in point-to-point contact with the strained elastomer, and its movement state is shown in Figure 1.

When the elastomer of the load cell is purely rolled, the lower indenter is fixed, and the upper indenter is translated in parallel with the transient of the contact point of the elastomer. Let point $\mathrm{A}$ and point $\mathrm{B}$ be the centre of the spherical surface before and after the lower ball end of the elastomer rolls, and point $\mathrm{C}$ be the centroid of the elastomer. According to the geometric relationship of the dimensions in the Figure 1 (a) and (b), among them has the same indenter and elastomer.

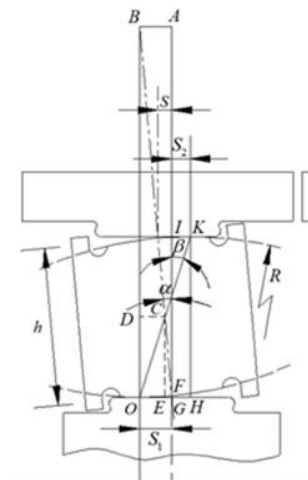

(a)

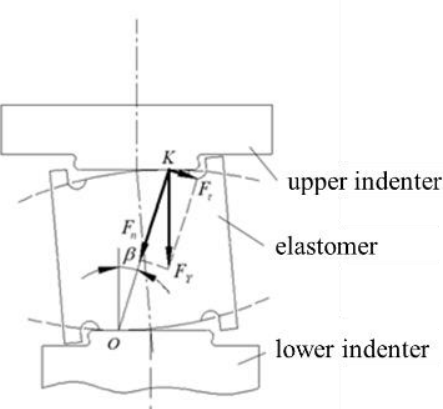

(b)
Figure 1: Oscillation support of the load cell, (a) Oscillation position change of the load cell, (b) Force analysis of the load cell 
In Figure $1, \alpha$ is the inclination angle of the sensor strain elastomer axis, $h$ is the height of the sensor strain elastomer along the axis, $\beta$ is the inclination angle of the connection point between the ball head and the upper indenter and the rolling point, $R$ is the radius of the ball head. $S$ is the horizontal displacement of the axis of the upper indenter. $S_{1}$ is the rolling displacement of the contact point between the ball head and the lower head. $S_{2}$ is the horizontal movement distance of the contact point between the ball head and the upper indenter.

For a stable and balanced oscillated support, the vertical force of the external load $F_{Y}$ on the elastomer at this time can be decomposed into a radial force $F_{n}$ and a tangential force $F_{\tau}$ along the rolling support point $\mathrm{O}$ of the elastomer, where the tangential force $F_{\tau}$ provides the restoring force promotes structural stability. When the system is oscillating slightly, tangential force can be expressed as follows:

$$
F_{\tau}=F_{Y} \sin \beta \approx \frac{(2 R-h) F_{Y}}{h} \alpha
$$

It can be known that the restoring force of the micro-oscillated support is approximately proportional to the oscillated angle and the vertical force applied.

\section{DYNAMIC MODEL OF OSCILLATED SUPPORT VIBRATION}

Low speed axle-group weigh-in-motion system has four column load cells as carriers. When the vehicle only travels along the longitudinal axis of the weighing platform, the scale body only vibrates in the longitudinal direction of the weighing platform, regardless of the lateral vibration of the weighing platform, and the movement form of the scale body is a single degree of freedom system. It can be seen from equation (1) that its swing stiffness is approximately proportional to the external load and offset angle of each sensor. The dynamic model of its oscillation support vibration is shown in Figure 2. Let $M_{O f i}(i=1,2,3,4)$ be the rolling friction couple of the elastomer and the lower pressure head. During the rolling process, the rolling friction resistance moment is approximately equal to the maximum rolling friction resistance moment $M_{\max }$, which is the vertical pressure $F_{O Y i}$ on the head bearing point is proportional to the size. Let the proportionality constant be $\delta$.

$M_{O f i} \approx M_{\max }=\delta F_{O Y i}$

The force applied to the weighing platform by the external load is simplified to the lateral force $F_{G S}$, the vertical force $F_{G}$ and the moment $M_{G f}$. The interaction between the weighing platform and the elastomer of the sensor can be regarded as a simply supported plate structure with point-to-surface contact. At the contact point $K$, the elastomer is only affected by the vertical force $F_{N i}$ of the weighing platform, the horizontal friction force $F_{X i}$ and the rolling friction couple $M_{K f i}$. We can also obtain:

$M_{K f i} \approx \delta F_{N i}$

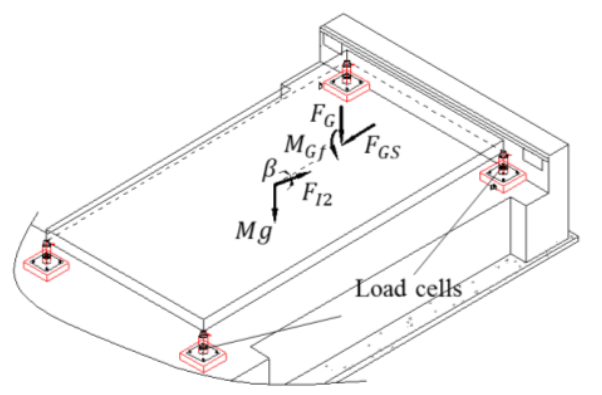

(a)

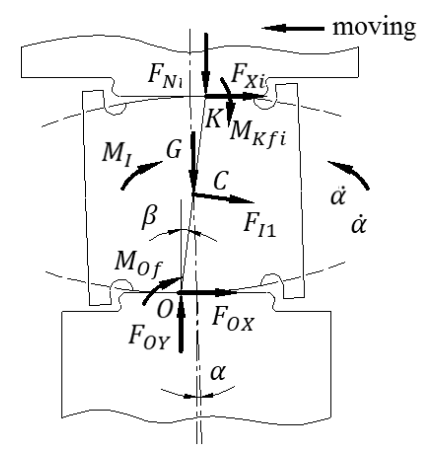

(b)

Figure 2: Dynamic model of vibration state of oscillation support, (a) Four-point support dynamic truck scale loaded model, (b) Force analysis model of column load sensor

The swing angle and direction of each column load cells are always consistent with the weighing platform. The total stiffness of the weighing system is equal to the sum of the swing stiffness of each parallel column load sensor. In the static state with the same swing angle, the stiffness of each column load cell is proportional to the vertical force. Letting the scale factor be $C$, we obtain:

$\frac{k_{i}\left(\alpha, F_{N i}\right)}{F_{N i}}=C \quad(i=1,2,3,4)$

$k=\sum_{i=1}^{4} k_{i}\left(\alpha, F_{N i}\right)=\sum_{i=1}^{4} C F_{N i}=C F_{N}$

It can be seen from formula (5) that when the vehicle is traveling on the weighing platform, although the force acting on each column load cell is transient, the support vibration of each sensor is synchronised with the vibration of the weighing platform, and the sum of the external forces received by the sensors remains unchanged. Taking four column load sensors as the research object, the column load cell takes the torque balance to the 
support point $O$ by the Lagrange's D'Alembert principle, and the other three load sensors are superimposed to balance the torque at each support point. After the equation, the dynamic control equation of the oscillation support vibration of the weighing system can be obtained:

$$
\begin{aligned}
M_{K f}+(M g+ & \left.F_{I 2} \sin \beta+F_{G}\right) \cdot O H \\
& +\left(F_{I 2} \cos \beta-F_{G S}\right) \cdot H K \\
& +M_{O f}+4 M_{I}+4 G \\
& \cdot O C \sin \beta+4 F_{I 1} \cdot O C=0
\end{aligned}
$$

where:

$$
\begin{aligned}
& M_{I}=J \ddot{\alpha} ; F_{I 1}=m \cdot O C \ddot{\beta} ; F_{I 2}=M \cdot O K \ddot{\beta} \\
& G=m g ; M_{K f}=\delta\left(M g+F_{I 2} \sin \beta+F_{G}\right) \\
& M_{O f}=\delta\left[M g+4 G+\left(4 F_{I 1}+F_{I 2}\right) \sin \beta+F_{G}\right]
\end{aligned}
$$

In these equations, $m$ is the mass of the elastomer, $M$ is the mass of the weighing platform, and $J$ is the rotational inertia of the elastomer.

According to the frictional moment of friction between the ball head and the upper and lower pressure heads, it always hinders the relative rolling of the elastomer, and the direction is opposite to the direction of the elastic angular speed of the elastomer. Static friction does not do work during pure rolling. Similar to the dissipation effect of Coulomb damping on the elastic potential energy of a spring oscillator, free vibration will gradually stabilize near the equilibrium position. Substituting the relevant parameters into equation (6), the vibration control equation of the system oscillation support is obtained:

$$
\begin{aligned}
& 2 \hat{\delta}[(1+2 \widehat{m}) \hat{g}+2(1+\widehat{m}) \cdot \hat{r} \cdot \hat{\hat{\beta}} \cdot \sin \beta+ \\
& \left.\widehat{F_{G}}\right]+\left(\hat{g}+\widehat{F_{G}}\right) \cdot \hat{l}-\widehat{F_{G S}} \cdot \hat{u}+4 \hat{J} \cdot \hat{\tilde{\alpha}}+4 \hat{m} \\
& \hat{g} \cdot \hat{r} \sin \beta+2[(\hat{l} \cdot \sin \beta+\hat{u} \cos \beta)+2 \widehat{m} . \\
& \hat{r}] \cdot \hat{r} \cdot \hat{\tilde{\beta}}=0 \quad(\hat{\dot{\beta}}>0)
\end{aligned}
$$

Among them:

$$
\begin{aligned}
& \widehat{m}=\frac{m}{M} ; \hat{r}=\frac{O C}{R} ; \hat{h}=\frac{h}{R} ; \hat{\delta}=\frac{\delta}{R} ; \hat{t}=\omega_{0} t \\
& \hat{g}=\frac{g}{R \omega_{0}^{2}} ; \hat{\jmath}=\frac{J}{M R^{2}} ; \hat{l}=\frac{O H}{R} ; \hat{u}=\frac{H K}{R} \\
& \widehat{F_{G}}=\frac{F_{G}}{M R \omega_{0}^{2}} ; \widehat{F_{G S}}=\frac{F_{G S}}{M R \omega_{0}^{2}} ; \hat{\ddot{\alpha}}=\frac{\ddot{\alpha}}{\omega_{0}^{2}} ; \hat{\hat{\beta}}=\frac{\ddot{\beta}}{\omega_{0}^{2}}
\end{aligned}
$$

\section{ANALYSIS OF AXLE LOAD CONDITIONS OF VEHICLES}

\subsection{Analysis of Axle Loading Conditions of Vehicles Running on the Road}

Suppose that the vehicle is driven by the rear axle. The axle type is composed of the front axle and the rear axle installed with two axles, and $F_{G 2}=$ $F_{G 3}$. The axle load and wheelbase are shown in Figure 3. It is assumed that when the vehicle is driving on the road surface, the front and rear wheels accelerate pure rolling motion under the action of the driving force. The driving wheel is subjected to driving torque, backward traction reaction force, vertical downward axle load, ground friction and rolling resistance. The traction force of the driven wheel is set forward, and the driven wheel is not affected by the driving torque.

Taking each axis as the research object, considering the tires belonging to a single axis as a whole, suppose the direction of each axis force and the direction of acceleration are shown in Figure 4 (a), (b), and the motion parameters of the two drive wheels are consistent, According to the direction of the static friction force of the pure rolling wheel in the figure, the magnitude of the static friction force is determined by the differential equation of motion.

$\left(10 m_{V}+M_{V}\right) a_{C}=2 F_{S 2}-F_{S 1}$

$2 J_{V} \ddot{\varphi}=-M_{f 1}+F_{S 1} R_{V}$

$a_{C}=R_{V} \ddot{\varphi}$

Among them:

$M_{f 1}=\delta^{\prime} F_{G 1} ; M_{f 2}=\delta^{\prime} F_{G 2}$

$M_{T}=M_{2}+M_{3}=2 M_{2} ; F_{n 1}+F_{n 2}+F_{n 3}=M_{V} g$

where $M_{V}$ is the body mass, $m_{V}$ is the mass of a single tire, $F$ is the traction force of the car to the front axle, $J_{V}$ is the tire moment of inertia, $\delta^{\prime}$ is the rolling friction coefficient between the wheel and the weighing platform, $R_{V}$ is the tire radius, $M_{T}$ is the total driving torque of the rear axle, $\ddot{\varphi}$ is the tire rolling angle acceleration.

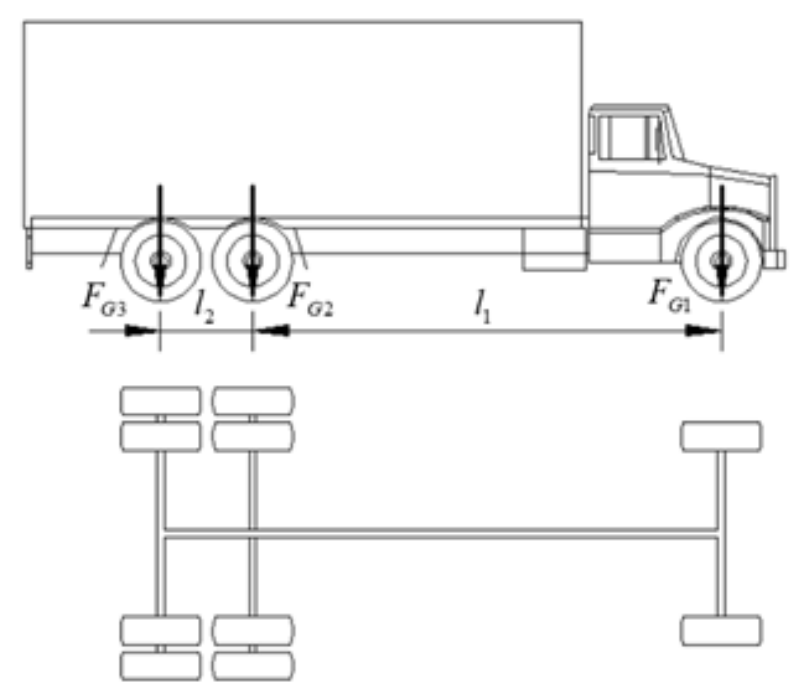

Figure 3: Schematic diagram of axle load and wheelbase of truck 


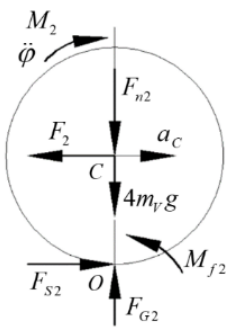

(a)

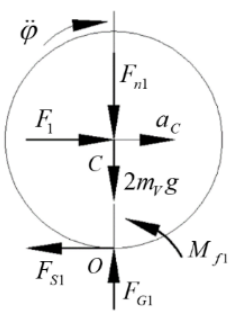

(b)

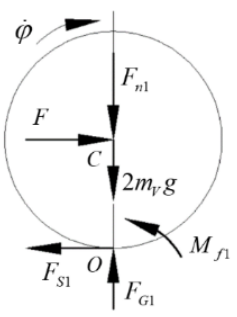

(c)
Figure 4: Schematic diagram of axle load and wheelbase of truck, (a) Rear axle drive wheel, (b) Front axle driven wheel, (c) Front axle rolls at a constant speed

We can obtain from equations (8) to (10):

$$
\begin{aligned}
& F_{S 1}=\frac{M_{f 1}}{R_{V}}+\frac{2 J_{V} a_{C}}{R_{V}^{2}} \\
& F_{S 2}=\frac{M_{f 1}}{2 R_{V}}+\left(5 m_{V}+0.5 M_{V}\right) a_{C}+\frac{J_{V} a_{C}}{R_{V}^{2}}
\end{aligned}
$$

Similarly, for the pure rolling state of uniform motion, as shown in Figure 4(c), the driving force is equal to the static friction force, that is $F=F_{S 1}$, which can be obtained from the balance relationship:

$$
F_{S 1}=2 F_{S 2}=\frac{M_{f 1}}{R_{V}}
$$

\subsection{Analysis of Axle Loading Conditions of Vehicles Running on Weighing Platform}

In order to determine the external disturbance force of the forced vibration of the column load cell oscillation support, the axle group type dynamic truck scale weighing system is selected as the research object. The four-point supported weighing platform performs variable speed movement under the impact of the vehicle, taking the vehicle's driving direction as the positive direction, and when the tire of a certain axis of the vehicle rolls purely on the weighing platform, the angular acceleration of its wheels is:

$$
\ddot{\varphi}^{\prime}=\frac{a_{C}-\ddot{S}}{R_{V}}
$$

The relationship between the tire rolling angle acceleration and the wheel centre translation acceleration of the other axles traveling on the road surface is the same as equation (10). In the same way, by balancing equations for each axis, the magnitude of the static friction force on each axis when the vehicle passes through the weighing platform can be obtained separately.

\section{NUMERIC CALCULATIONS}

In order to obtain the vibration characteristics of the swing load of the column load sensor, the response of the column load cell is selected as the research object under the dynamic weighing of the axle group dynamic truck scale. The free vibration of the oscillation support of the weighing system and the forced vibration when the vehicle is under dynamic load are selected for simulation when there is no vehicle running.

\subsection{Numerical Simulation of Free Vibration}

Considering that the ball radius of the elastomer affects the weighing accuracy of the eccentric load of the column load sensor, only the influence of the adjustable elastomer height on the vibration state of the oscillation support is analysed here. In order to eliminate other influencing factors, the oscillation support is set as free vibration, which is not affected by the vehicle load, that is, $F_{G}, F_{G S}$, and $M_{G f}$ are all zero. Select the load cell parameters of the column load cell C16A C3 operating manual with a maximum range of $20 \mathrm{t}$. Suppose the rolling friction coefficient of the elastomer and the upper and lower indenters is according to the coefficient between the steel wheel and the rail given by theoretical mechanics, and the relevant parameters are shown in Table 1.

Due to $M$ being much larger than $m$, when the angle $\alpha$ is small, the mass and rotational inertia of the elastomer of the load cell can be ignored, and $\sin \alpha \approx \alpha, \cos \alpha \approx \cos \beta=1$. It can be seen from Figure 5 that as the height of the elastomer decreases, the time for the swing load vibration of the column load cell to reciprocate for one week decreases, the amplitude increases, and the vibration attenuation time also increases.

Table 1: Free vibration parameters of oscillation support

\begin{tabular}{|c|c|c|}
\hline Parameter & Value & Unit \\
\hline$m$ & 2.5 & $\mathrm{~kg}$ \\
\hline$R$ & 130 & $\mathrm{~mm}$ \\
\hline$h$ & 150 & $\mathrm{~mm}$ \\
\hline$\delta$ & 0.05 & $\mathrm{~mm}$ \\
\hline$M$ & 4860 & $\mathrm{~kg}$ \\
\hline$J$ & $4.59 \times 10^{-3}$ & $\mathrm{~kg} \cdot \mathrm{m}^{2}$ \\
\hline
\end{tabular}

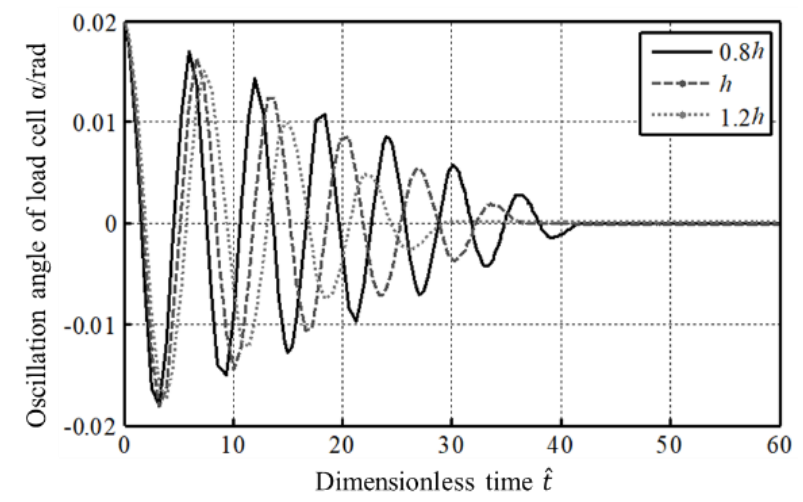

Figure 5: Numerical simulation curve of free vibration of oscillation support 


\subsection{Numerical Simulation of Forced Vibration of Oscillation support in Low Speed Weighing}

When forced to vibrate, suppose the weighing system is in equilibrium, and the initial angular displacement $\alpha_{0}$ and initial angular velocity are both zero. The Dongfeng DFL1250A6 cargo truck (type 1+5) is selected as the rear-wheel drive vehicle with axle load. The rear axle is a dual-axle mounted side by side. The axle load and wheelbase are shown in Figure 3. The wheel tires are radial wide rim tires and tires. The specification is $10.00 \mathrm{R} 20$, and the tire radius is $R_{V}=527 \mathrm{~mm}$. The rolling friction coefficient between the wheel and the scale surface is taken as the middle value $\delta^{\prime}=6 \mathrm{~mm}$, and other relevant parameters are shown in Table 2.

Table 2: Force vibration parameters of oscillation support

\begin{tabular}{|c|c|c|}
\hline Parameter & Value & Unit \\
\hline$m_{V}$ & 70 & $\mathrm{~kg}$ \\
\hline$J_{V}$ & 7.56 & $\mathrm{~kg} \cdot \mathrm{m}^{2}$ \\
\hline$F_{G 1}$ & 68000 & $\mathrm{~N}$ \\
\hline$F_{G 2}$ & 89000 & $\mathrm{~N}$ \\
\hline$F_{G 3}$ & 89000 & $\mathrm{~N}$ \\
\hline$l_{1}$ & 4350 & $\mathrm{~mm}$ \\
\hline$l_{2}$ & 1300 & $\mathrm{~mm}$ \\
\hline
\end{tabular}

Assuming that the vehicle drives through the weighing platform at a constant speed of $v=2 \mathrm{~m} \cdot \mathrm{s}^{-1}, a_{C}=0 \mathrm{~m} \cdot \mathrm{s}^{-2}$, the axle load and horizontal friction force of each axis of the vehicle acting on the weighing platform at different time periods are almost constant. The following only solves the axle load driving on the weighing platform, and the angular acceleration of the wheels driving on the weighing platform can be obtained from equation (14):

$\ddot{\varphi}^{\prime}=-\frac{\ddot{S}}{R_{V}}$

From the static friction formula (13) and formula (15) of constant speed rolling, we can obtain static friction force when the front axle travels on the weighing platform:

$F_{S 1}^{\prime}=F^{\prime}=\frac{M_{f 1}}{R_{V}}-\frac{2 J_{V} \ddot{S}}{R_{V}^{2}}$

When the front axle is on the weighing platform, the static friction of the rear axle is:

$F_{S 2}^{\prime}=F_{S 3}^{\prime}=\frac{F^{\prime}}{2}$

The length of the weighing platform is $5250 \mathrm{~mm}$, and the driving process of the vehicle on the weighing platform can be obtained according to the vehicle speed and the wheelbase. When the vehicle is just on the scale, set $t=0 \mathrm{~s}$, then only the front axle of the weighing platform applies load on the weighing platform, and the time interval until the second axis applies the load is:

$t=\frac{l_{1}}{v}=\frac{4.35}{2}=2.175 \mathrm{~s}$

The total vertical force applied to the weighing platform $F_{G}=F_{G 1}$, the total friction force is given by equation (16) $F_{G S}=F_{S 1}$, and rolling friction couple $M_{G f}=\delta^{\prime} F_{G 1}$. Similarly, the change process of the axle load applied to the weighing platform in the remaining time period can be obtained, as shown in Table 3.

Table 3: Loading conditions of weighing platform in different time periods

\begin{tabular}{|c|c|c|c|}
\hline Time $/ \mathbf{s}$ & $\boldsymbol{F}_{\boldsymbol{G}}$ & $\boldsymbol{F}_{\boldsymbol{G S}}$ & $\boldsymbol{M}_{\boldsymbol{G} \boldsymbol{f}}$ \\
\hline $0.000-2.175$ & $F_{G 1}$ & $F_{S 1}^{\prime}$ & $\delta^{\prime} F_{G 1}$ \\
\hline $2.175-2.625$ & $F_{G 1}+F_{G 2}$ & $F_{S 1}^{\prime}-F_{S 2}^{\prime}$ & $\begin{array}{c}\delta^{\prime}\left(F_{G 1}\right. \\
\left.+F_{G 2}\right)\end{array}$ \\
\hline $2.625-2.825$ & $F_{G 2}$ & $-\bar{F}_{S 2}$ & $\delta^{\prime} F_{G 2}$ \\
\hline $2.825-4.800$ & $F_{G 2}+F_{G 3}$ & $\begin{array}{c}-\bar{F}_{S 2} \\
-\bar{F}_{S 3}\end{array}$ & $\begin{array}{c}\delta^{\prime}\left(F_{G 2}\right. \\
\left.+F_{G 3}\right)\end{array}$ \\
\hline $4.800-5.450$ & $F_{G 3}$ & $-\bar{F}_{S 3}$ & $\delta^{\prime} F_{G 3}$ \\
\hline$>5.450$ & 0 & 0 & 0 \\
\hline
\end{tabular}

Similarly, considering $M$ is much larger than $m$, when the angle $\alpha$ is small, the mass and moment of inertia of the sensor elastomer can be ignored. Substituting the above given parameters, the initial angular displacement $\alpha_{0}$ and initial angular velocity of the oscillation support vibration are both zero, and the forced vibration solution of the car at a constant speed is shown in Figure 6. It can be seen from Figure 6 that with the increase of the shaft load, the more significant the vibration of the column load cell oscillation support, the faster the reciprocating motion, the greater the amplitude, and the longer the vibration attenuation time. It can be seen from Figure 7 that increasing the flatness of the weighing platform surface and reducing the rolling friction couple to reduce the horizontal static friction between the wheel and the weighing platform can significantly reduce the amplitude of the oscillation support vibration, because the horizontal static friction is much less than the vertical to gravity, the time of one round of reciprocation is almost unchanged, and the decay time of vibration is also shorter. 


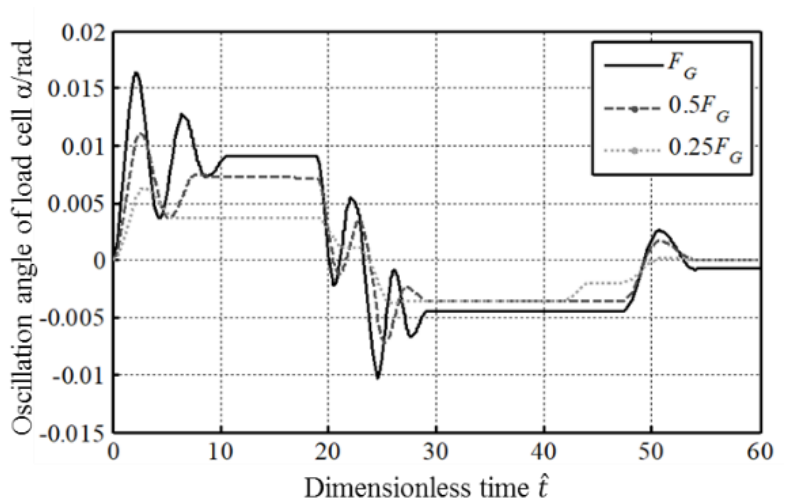

Figure 6: Numerical simulation curve of forced vibration at constant speed driving

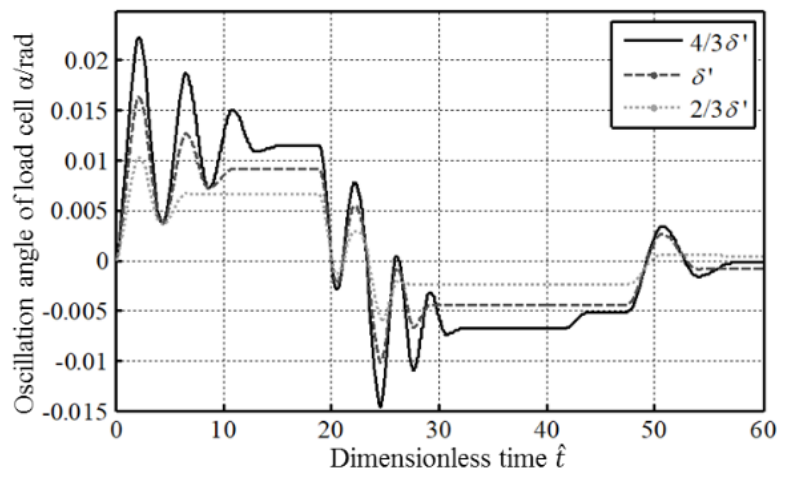

Figure 7: Numerical simulation curve of forced vibration at acceleration state

\section{SUMMARY}

The oscillation support column load cell shows vibration characteristics during dynamic weighing, which affects the stability and accuracy of weighing, and even affects the fatigue life of the load cell. The conclusions of this study can be used to analyse the dynamic process of the vibration of the load cell oscillation support and the influence of the weight accuracy, and can provide a basis for the corresponding vibration control or vibration reduction design. It can be concluded that:

(1) The supporting vibration restoring force is approximately proportional to the vibration angular displacement and load capacity.

(2) The recovery time of the oscillation support vibration of the weighing system decreases as the height of the elastomer decreases, the amplitude increases as the height of the elastomer decreases, and the vibration attenuation time increases as the height of the elastomer decreases. The noise interference frequency generated by the horizontal shaking of the scale body will affect the weighing data processing. Under the condition of weighing accuracy, the height of the elastomer can be appropriately increased to reduce the vibration swing rate and amplitude.

(3) The vibration stiffness of the horizontal support of the weighing platform is related to the magnitude of the load on the weighing platform, and has nothing to do with the distribution of the load. With the increase of the axial load, the vibration recovery time decreases, the amplitude increases, the more significant the vibration, the longer the vibration attenuation time. In general, the limit device is used to limit and consume the vibration of the weighing platform.

(4) Under the condition of anti-skid, the flatness of the weighing platform surface should be improved, and the rolling friction resistance should be reduced to reduce the horizontal static friction between the wheel and the weighing platform. The amplitude of the oscillation support vibration can be significantly reduced, and the vibration recovery time is almost change, and the faster the vibration decay.

\section{REFERENCES}

[1] D. Rys, J. Judycki, P. Jaskula, “Analysis of effect of overloaded vehicles on fatigue life of flexible pavements based on weigh in motion (WIM) data", International Journal of Pavement Engineering, vol. 17, no. 8, pp. 716-726, 2016.

[2] Xianzhi Dai, "An vibration energy harvester with broadband and frequency-doubling characteristics based on rotary pendulums", Sensors and Actuators A: Physical, vol. 241, pp. 161-168, 2016.

[3] M. C. Ciornei, S. Alaci, F. C. Ciornei et al, "A method for the determination of the coefficient of rolling friction using cycloidal pendulum", IOP Conference Series: Materials Science and Engineering, vol. 227, no. 1, pp. 12-27, 2017.

[4] I. Gilavdary, S. Mekid, N. Riznookaya, "Microslippage effects in pre-rolling induced by a disturbed and undisturbed pendulum with spherical supports", Proceedings of the Institution of Mechanical Engineers Part J Journal of Engineering Tribology, vol. 228, no. 1, pp. 46-52, 2014. 